\title{
Charged-Current Interaction Measurements in MiniBooNE
}

\author{
Teppei Katori for the MiniBooNE collaboration
}

Indiana University, Bloomington, IN

\begin{abstract}
Neutrino oscillation is the only known phenomenon for physics beyond the standard model. To investigate this phenomenon, the understanding of low energy neutrino scattering $(200<$ $\left.E_{v}<2000 \mathrm{MeV}\right)$ is the crucial task for high energy physicists. In this energy region, the charged current quasi-elastic (CCQE) neutrino interaction is the dominant process, and experiments require a precise model to predict signal samples. Using a high-statistics sample of muon neutrino CCQE events, MiniBooNE finds that a simple Fermi gas model, with appropriate adjustments, accurately characterizes the CCQE events on carbon. The extracted parameters include an effective axial mass, $M_{A}=1.23 \pm 0.20 \mathrm{GeV}$, and a Pauli-blocking parameter, $\kappa=1.019 \pm 0.011$.
\end{abstract}

Keywords: axial mass, charged current quasi-elastic, neutrino, MiniBooNE, Pauli blocking PACS: $11.80 . \mathrm{Cr}, 13.15 .+\mathrm{g}, 14.60 . \mathrm{Lm}, 14.60 . \mathrm{Pq}$

\section{CCQE event selection in MiniBooNE}

The MiniBooNE detector, a spherical tank filled with mineral oil, detects Čerenkov light from charged particles 1 . The identification of $v_{\mu} \mathrm{CCQE}$ interactions relies solely on the detection of the primary muon Čerenkov light and the associated decay electron Čerenkov light in these events (Fig. (1):

$$
v_{\mu}+n \rightarrow \mu^{-}+p \quad \mu^{-} \rightarrow e^{-}+v_{\mu}+\bar{v}_{e} .
$$

By avoiding requirements on the outgoing proton kinematics, the selection is less dependent on nuclear models. The scintillation light from the proton, although not used directly in the $v_{\mu} \mathrm{CCQE}$ analysis, is intensively studied in neutral current elastic scattering events at MiniBooNE [3]. A total of 193,709 events pass the MiniBooNE $v_{\mu}$ CCQE selection criteria [2] from $5.58 \times 10^{20}$ protons on target collected between August 2002 and December 2005. The cuts are estimated to be $35 \%$ efficient at selecting $v_{\mu}$ CCQE events in a $500 \mathrm{~cm}$ radius, with a CCQE purity of $74 \%$. The predicted backgrounds are: $74.8 \%$ CC $1 \pi^{+}, 15.0 \%$ CC $1 \pi^{0}, 4.0 \%$ NC $1 \pi^{ \pm}, 2.6 \%$ CC multi- $\pi, 0.9 \%$ NC elastic, $0.8 \% \bar{v}_{\mu} \mathrm{CC} 1 \pi^{-}, 0.8 \% \mathrm{NC} 1 \pi^{0}, 0.6 \% \eta / \rho / K$ production, and $0.5 \%$ deep inelastic scattering and other events. Because pions can be absorbed via final state interactions in the target nucleus, a large fraction of the background events look like CCQE events in the MiniBooNE detector. "CCQE-like" events, all events with a muon and no pions in the final state, are predicted to be $84 \%$ of the sample after cuts.

\footnotetext{
${ }^{1}$ The detailed information of the Fermilab Booster neutrino beamline and the MiniBooNE neutrino detector are available elsewhere [1, 2].
} 


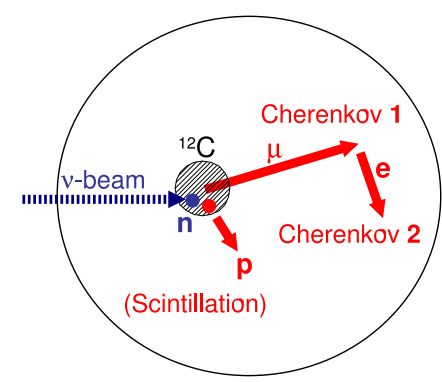

FIGURE 1. Schematic figure of a CCQE interaction. The primary Čerenkov light from the muon (Čerenkov 1, first subevent) and subsequent Čerenkov light from the decayed electron (Čerenkov 2, second subevent) are used to tag the CCQE event. For most events, protons only emit scintillation light, and our selection is insensitive to this information.

\section{MiniBooNE CCQE events}

Fig 2 shows the data-Monte Carlo (MC) ratio of CCQE events as a function of muon kinetic energy $T_{\mu}(\mathrm{GeV})$ and muon scattering angle $\cos \theta_{\mu}$. Note the muon energy and muon scattering angle are the observables and the basis of all reconstructed kinematic variables in the $v_{\mu} \mathrm{CCQE}$ channel. One can immediately see that the data-MC agreement is poor. There are 6 auxiliary lines: (a), (b), and (c) are equal neutrino energy lines, 0.4 , 0.8 , and $1.2 \mathrm{GeV}$ each, and (d), (e), and (f) are equal $Q^{2}$ lines, $0.2,0.6$, and $1.0 \mathrm{GeV}^{2}$ each. The data-MC disagreement follows auxiliary lines of equal $Q^{2}$, not equal neutrino energy, this indicates that the data-MC disagreement is not due to the neutrino flux prediction, but due to the neutrino interaction prediction, because the former is a function of neutrino energy and the latter is a function of $Q^{2}$. So we assume that the data-MC disagreement comes from our neutrino interaction model and we adjust to the data. This is a critical task for MiniBooNE since the goal is to measure $v_{e} \mathrm{CCQE}$ events, but MC and all reconstruction tools must be reliable and tested in copious $v_{\mu} \mathrm{CCQE}$ events due to the blind analysis constraint on the $v_{e} \mathrm{CCQE}$ channel.

The data-MC disagreement is classified in 2 regions in this plane (Fig. 2),

1. data deficit at low $Q^{2}$ region, light gray band near the top left corner

2. data excess at high $Q^{2}$ region, black band from the top right to the bottom left

Since we are employing the Relativistic Fermi Gas (RFG) model [4] in our MC, we wish to fix these problems within the RFG model. The low $Q^{2}$ physics is usually controlled by nuclear model, so we want to tune the nuclear model, especially the strength of Pauli blocking, to fix region (1). This is justified because electron scattering data has not provided precise information for Pauli blocking in the low $Q^{2}$ region in terms of the RFG model [5]. For (2), we need to increase axial mass $M_{A}$ to increase the cross section at high $Q^{2}$. Here, the axial mass is understood as an experimental parameter in the axial form factor,

$$
F_{A}\left(Q^{2}\right)=\frac{g_{A}}{\left(1+\frac{Q^{2}}{M_{A}^{2}}\right)^{2}}
$$




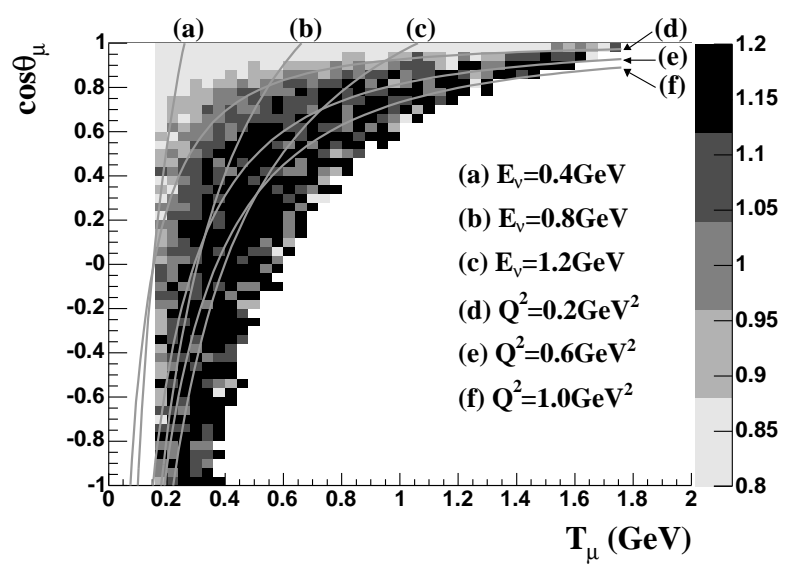

FIGURE 2. Ratio of MiniBooNE $v_{\mu}$ CCQE data/simulation as a function of reconstructed muon angle and kinetic energy. The prediction is prior to any CCQE model adjustments; the $\chi^{2} /$ dof $=79.5 / 53$. The ratio forms a $2 \mathrm{D}$ surface whose values are represented by the gray scale, shown on the right. If the simulation modeled the data perfectly, the ratio would be unity everywhere. Contours of constant $E_{V}$ and $Q^{2}$ are overlaid.

where $g_{A}$ is axial coupling constant $(=1.267)$. This treatment is also justified because elastic electron scattering cannot measure the axial mass precisely. Interestingly, the high axial mass is also observed by the $\mathrm{K} 2 \mathrm{~K}$ experiment in Japan [6].

\section{Pauli blocking parameter $\kappa$}

Currently, MiniBooNE is using the NUANCE neutrino interaction generator [7]. In NUANCE, CCQE interactions on carbon are modeled by the Relativistic Fermi Gas (RFG) model [4]. To achieve our goal within the RFG model, we introduced a new parameter "kappa", $\kappa$,

$$
E_{\mathrm{hi}}=\sqrt{p_{F}^{2}+M_{n}^{2}} \quad E_{\mathrm{lo}}=\kappa\left(\sqrt{p_{F}^{2}+M_{p}^{2}}-\omega+E_{B}\right)
$$

where $M_{n}$ is the target neutron mass, $M_{p}$ is the outgoing proton mass, $P_{F}$ is Fermi momentum $(=220 \mathrm{MeV}), E_{B}$ is binding energy $(=34 \mathrm{MeV})$, and $\omega$ is the energy transfer. In the RFG model, $E_{\mathrm{hi}}$ is the energy of an initial nucleon on the Fermi surface and $E_{\mathrm{lo}}$ is the lowest energy of an initial nucleon that leads to a final nucleon just above the Fermi momentum. The function of parameter $\kappa$ is to squeeze down the phase space of the nucleon Fermi sea, especially when the energy transfer is small. From Fig. 3 , one can see that this parameter controls the $Q^{2}$ distribution only in the low $Q^{2}$ region. This is quite complementary to the role of $M_{A}$, since $M_{A}$ mainly controls the $Q^{2}$ distribution in the high $Q^{2}$ region.

We use these 2 parameters to perform a grid search to find the $\chi^{2}$ minimum. Here, we take into account all possible correlations between systematics by using the inverse of the full error matrix, not by adding systematics as pull terms. 

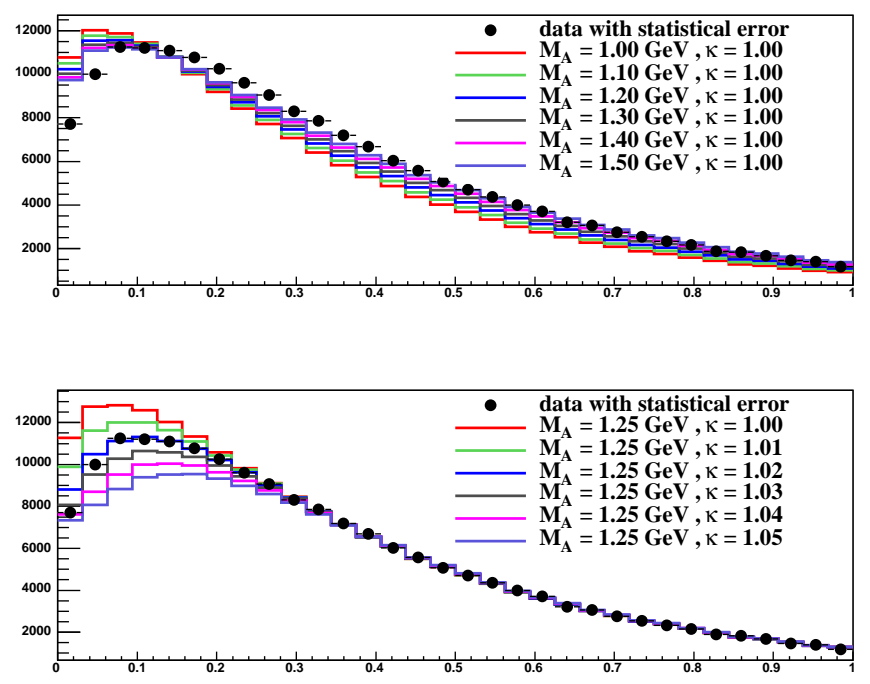

FIGURE 3. Effect of $M_{A}$ and $\kappa$ variations on the MiniBooNE, flux integrated $Q^{2}$ distribution. The top plot shows various $M_{A}$ with fixed $\kappa$, and bottom plot shows various $\kappa$ with fixed $M_{A}$. Note, the $M_{A}$ variation has large impact at high $Q^{2}$ while the $\kappa$ variation has a significant impact only for $Q^{2}$ below $\sim 0.2 \mathrm{GeV}^{2}$.

\section{Fit result}

Finally, the parameters extracted from the MiniBooNE $v_{\mu}$ CCQE data are:

$$
\begin{aligned}
M_{A} & =1.23 \pm 0.20 \mathrm{GeV} \\
\kappa & =1.019 \pm 0.011
\end{aligned}
$$

Tab. 1 shows the contributions to the systematic uncertainties on $M_{A}$ and $\kappa$. The detector model uncertainties dominate the error in $M_{A}$ due to their impact on the energy and angular reconstruction of CCQE events in the MiniBooNE detector. The dominant error on $\kappa$ is the uncertainty in the $Q^{2}$ shape of background events.

The result of this fitting, including all sources of systematic uncertainty, is shown in Fig. 4. Since the background error dominates at low $Q^{2}$, and it drives the large error bars at low $Q^{2}$. Note that, the shape uncertainty of the background, namely the $Q^{2}$ distribution shape uncertainty of $C C 1 \pi^{+}$events, is not included in these error bands. From the data, we know that the predicted $Q^{2}$ shape of $C C 1 \pi^{+}$events have large errors [8]. The extracted shape information from the data is implemented in our MC, and the fit is performed again. The result of those 2 fits, one using the MC predicted $C C 1 \pi^{+}$distribution, and the other is using MC tuned on $C C 1 \pi^{+}$data, are shown with the star and the triangle in the inserted plot in Fig. 4. The difference is interpreted as a background shape uncertainty error and added to the extracted parameters.

Fig. 5 shows the agreement between data and simulation after incorporating the $M_{A}$ and $\kappa$ values from the $Q^{2}$ fit to MiniBooNE $v_{\mu} \mathrm{CCQE}$ data. Comparing to Fig. 2 , the 


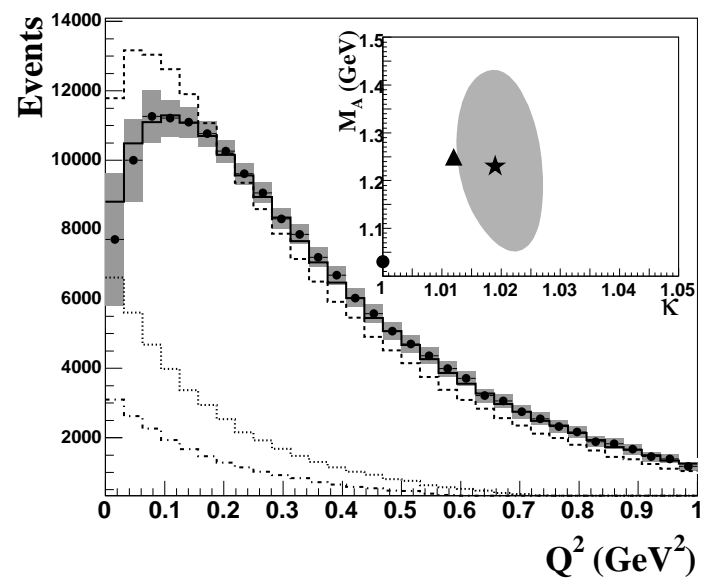

FIGURE 4. Reconstructed $Q^{2}$ for $v_{\mu} \mathrm{CCQE}$ events including systematic errors. The simulation, before (dashed) and after (solid) the fit, is normalized to data. The dotted (dot-dash) curve shows backgrounds that are not CCQE (not "CCQE-like"). The inset shows the $1 \sigma$ CL contour for the best-fit parameters (star), along with the starting values (circle), and fit results after varying the background shape (triangle).

TABLE 1. Uncertainties in $M_{A}$ and $\kappa$ from the fit to MiniBooNE $v_{\mu}$ CCQE data. The total error is not a simple quadrature sum because of the correlation between the two parameters.

\begin{tabular}{ccc}
\hline error source & $\delta M_{A}$ & $\delta \kappa$ \\
\hline data statistics & 0.03 & 0.003 \\
neutrino flux & 0.04 & 0.003 \\
neutrino cross sections & 0.06 & 0.004 \\
detector model & 0.10 & 0.003 \\
$\mathrm{CC} \pi^{+}$background shape & 0.02 & 0.007 \\
\hline total error & 0.20 & 0.011 \\
\hline
\end{tabular}

improvement is substantial and the data are well-described throughout the kinematic phase space. Since the whole kinematic space is fixed, not surprisingly, all of the individual kinematic variables exhibit good data-MC agreement. Fig. 6 shows that data and $\mathrm{MC}$ agree well within error bars for reconstructed muon neutrino energy and muon scattering angle.

In general, varying $M_{A}$ allows us to reproduce the high $Q^{2}$ behavior of the observed data events. A fit for $M_{A}$ above $Q^{2}>0.25 \mathrm{GeV}^{2}$ yields consistent results, $M_{A}=1.25 \pm 0.12 \mathrm{GeV}$ (Fig. 7). However, fits varying only $M_{A}$ across the entire $Q^{2}$ range leave considerable disagreement at low $Q^{2}$. This data-MC disagreement at low $Q^{2}$ would eventually reflect in data-MC disagreement in reconstructed neutrino energy, because data-MC disagreement in $Q^{2}$ spreads out in the kinematic plane and would affect the energy reconstruction across a wide region. The Pauli-blocking parameter $\kappa$ is instrumental here, enabling this model to match the behavior of the data down to $Q^{2}=0$. 


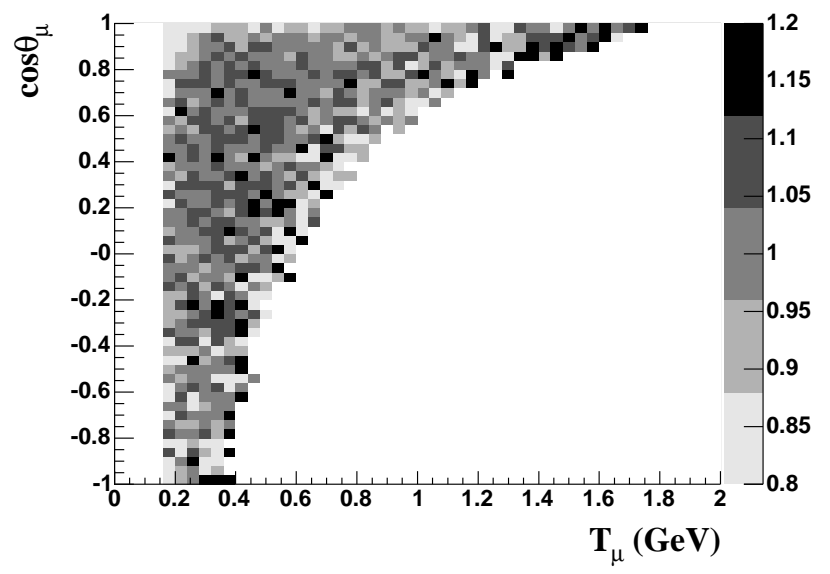

FIGURE 5. Ratio of data/simulation as a function of muon kinetic energy and angle after the CCQE model adjustments; the $\chi^{2} / \mathrm{dof}=45.1 / 53$. Compare to Figure 2
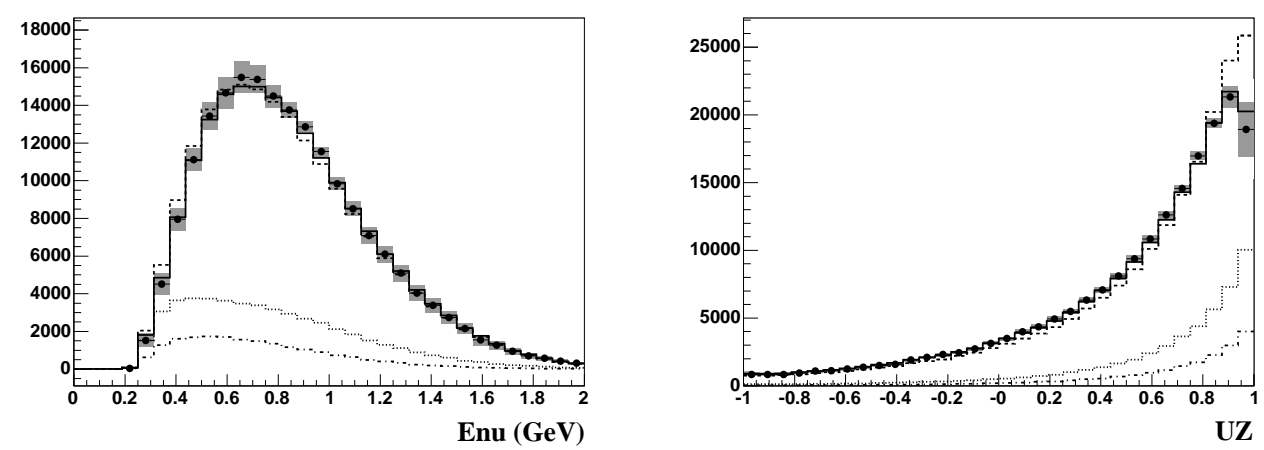

FIGURE 6. Reconstructed muon neutrino energy and measured muon scattering angle, line notations are the same as Fig 4
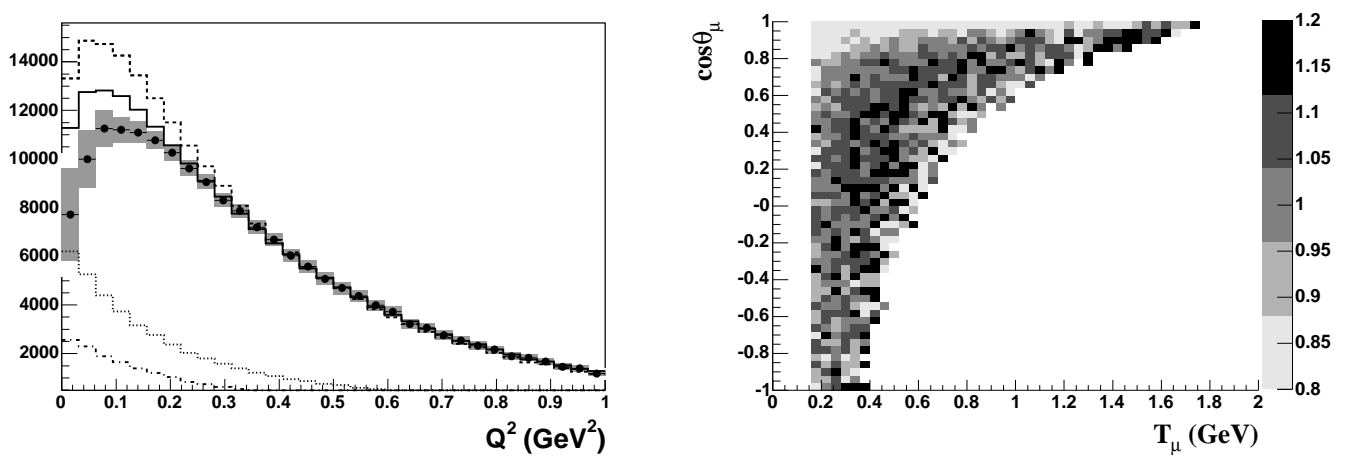

FIGURE 7. Reconstructed $Q^{2}$ for $v_{\mu} \mathrm{CCQE}$ events and data-MC ratio in the kinematic plane. The left plot is the analogy of Fig. 4 and the right plot is the analogy of Fig. 5but the fit is performed using $M_{A}$ only, with fixed $\kappa(=1.0$, no enhanced Pauli blocking $)$. 


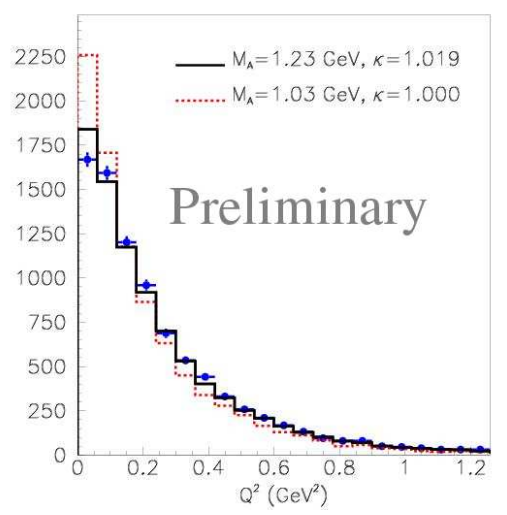

FIGURE 8. Reconstructed $Q^{2}$ for $\bar{v}_{\mu} \mathrm{CCQE}$ events, including statistics error only. The simulation, old model (dashed) and new model (solid) is normalized to data.

\section{Anti-neutrino CCQE preliminary result}

Finally, we tested the modified RFG model in a new sample of MiniBooNE antineutrino data (for other preliminary results for antineutrino run, see [9]). If our assumption is correct, this RFG model should also succeed in modeling $\bar{v}_{\mu}$ CCQE events. The result is shown in Fig. 8. Although statistics are low, one can tell the new model will describe the features of this data better than the original model.

In summary, taking advantage of the high-statistics MiniBooNE $v_{\mu}$ CCQE data, we have extracted values of an effective axial mass parameter, $M_{A}=1.23 \pm 0.20 \mathrm{GeV}$, and a Pauli-blocking parameter, $\kappa=1.019 \pm 0.011$, achieving substantially improved agreement with the observed kinematic distributions in this data set.

The $M_{A}$ value reported here should be considered an "effective parameter" in the sense that it may be incorporating nuclear effects not otherwise included in the RFG model. Future efforts will explore how the value of $M_{A}$ extracted from the MiniBooNE data is altered upon replacement of the RFG model with more advanced nuclear models [10].

\section{REFERENCES}

1. A. A. Aguilar-Arevalo et al. [MiniBooNE Collaboration], Phys. Rev. Lett. 98, 231801 (2007); R. Tayloe, reference in these proceedings

2. A. A. Aguilar-Arevalo et al. [MiniBooNE Collaboration], submitted to PRL [arXiv:0706.0926]

3. D. C. Cox, reference in these proceedings

4. R. A. Smith and E. J. Moniz, Nucl. Phys. B43, 605 (1972); erratum: ibid. B101, 547 (1975)

5. A. V. Butkevich and S. P. Mikheyev, Phys. Rev. C72, 025501 (2005)

6. R. Gran et al. [K2K collaboration], Phys. Rev. D74, 052002 (2006)

7. D. Casper, Nucl. Phys. Proc. Suppl. 112, 161 (2002)

8. B. T. Fleming, reference in these proceedings

9. V. Nguyen, reference in these proceedings

10. J. E. Amaro et al., Phys. Rev. C71, 015501 (2005); T. Leitner et al., Phys. Rev. C73, 065502 (2006); O. Benhar et al., Phys. Rev. D72, 053005 (2005); S. Ahmad et al., Phys. Rev. D74, 073008 (2006) 